\title{
Analysis of Coupled Surface Plasmon in LHM Mediated Dielectric Gap Multilayer Structure
}

\author{
Mohammed BENDJEBBOUR, Abdellatif CHERIFI, and Benamar BOUHAFS* \\ University of Tlemcen, Faculty of Sciences, Theoretical Physics Laboratory, Tlemcen 13000, Algeria \\ *Corresponding author: Benamar BOUHAFS $\quad$ E-mail: bouhafs_ben@yahoo.fr
}

\begin{abstract}
The purpose of this work is to investigate theoretically the characteristics of confined electromagnetic modes propagating along the interfaces of a multilayer device. This one dimensional (1D) sensor is formed by stacking a left-handed material (LHM) layer between a $\mathrm{SiO}_{2}$-glass prism and a dielectric gap layer in contact with gold $(\mathrm{Au})$. The results indicate that the total thickness of the LHM layer and dielectric gap, in optimum conditions, give the ability of tuning significantly the characteristics of the resonant modes correlated to surface plasmons (SPs) propagation along the interfaces of the designed device. By considering two arrangements between LHM and Au, two opposite resonant behaviors observed in p-reflectance spectra are analyzed in the angular interrogation mode and discussed thoroughly.
\end{abstract}

Keywords: Confined electromagnetic modes; surface plasmon resonance sensor; left-handed material

Citation: Mohammed BENDJEBBOUR, Abdellatif CHERIFI, and Benamar BOUHAFS, "Analysis of Coupled Surface Plasmon in LHM Mediated Dielectric Gap Multilayer Structure,” Photonic Sensors, 2020, 10(2): 113-122.

\section{Introduction}

Recently, metal-dielectric multilayer nanostructures supporting the propagation of surface plasmons (SPs) in appropriate conditions are currently investigated in the interest to assess particularly the limit of their specific sensitivity enhancement [1-3]. Generally, this performance parameter, which is requested to be high, is evaluated on the shift of the resonance condition with respect to any changes introduced in the refractive index (RI) of a sensing medium [4]. Generally, an interface performed between an infinite active metallic layer, such as silver (Ag), gold $(\mathrm{Au})$, aluminum $(\mathrm{Al})$, and a dielectric medium, once analyzed with p-polarized light beam via the attenuated total reflection (ATR) technique, exhibits an SP propagation. It should be noted that the angular resonance condition, of the generated SP mode, depends critically on both RIs and thicknesses of the whole associated media of the designed multilayer configuration [5-7]. Furthermore, the shift produced on the resonance peak and its line width evaluated on an interfacial SP profile were adopted as references to highlight the temperature effect [8], the carriers concentration of doped Si [9], percentages in nano-composite materials [10], the control of interface stability [11], and so on. Based on both the changes in resonance angles, $\theta_{1}=\theta_{\mathrm{SPR}}$ of the reflectance spectrum and its width, numerous types of geometries with appropriate choices of active materials have been reported in [7, 9, 12-15] and applied to wide uses such as gas detection [16, 17], medical diagnosis [18], and photonic devices [19]. By the use of graphene multilayer/Ag, Verma et al. [20] and

Received: 19 October 2018 / Revised: 28 May 2019

(C) The Author(s) 2019. This article is published with open access at Springerlink.com

DOI: $10.1007 / \mathrm{s} 13320-019-0568-3$

Article type: Regular 
Szunerits et al. [6] who also employed other strategies, succeeded in designing highly sensitive SPR sensors. Generally, in the design of SPR sensors, the sensing properties may be tuned in different limits that depend mainly on the whole of the parameters of involved active materials and the surrounding dielectrics [20-24]. In order to further improve the relative sensitivity, Ouyang et al. [25] used the advantages of stacking $\mathrm{Si} / \mathrm{MoS}_{2}$ under the $\mathrm{Au}$ layer exhibiting a single SPR mode whose full width at half maximum (FWHM) termed, $d q_{0.5}$ at $50 \%$ of reflectivity, is significantly wider when increasing the number of $\mathrm{MoS}_{2}$ layers. To produce a sharp and sensitive peak ascribed to coupled modes, Kullab et al. [26] proposed a novel four-layer scheme where a metamaterial (left-handed material and right-handed material) is included as a core layer to demonstrate that the designed structure presents an advantage over other optical sensors. It should be noted that the largest peak of the resonance condition, measured on angular reflectance spectra, constitutes a disadvantage for using the resonance angle as a key parameter in characterizing RI of aqueous solutions on the SPR sensors' surface $[27,28]$.

To manipulate the sensitivity in acceptable limits, recent research [29] adopted the strategies including the porosity effect in an SPR sensor to estimate the angular SPR curve and detect the accuracy (D.A $=1 / \Delta \theta_{0.5}$ ) of $1.098 /{ }^{\circ}$. In order to maximize the sensing sensitivity, a slab waveguide structure, based on the air gap enclosed between anisotropic LHM layers operating in the microwave frequency range, has been explored for refractometry applications [30].

Here, in the purpose of improving the sensitivity in a different way, we investigate the sensing response of a planar multilayer structure disposed on a prism-coupler. We discuss the influence on both RI and the thickness of an inner core (sensing medium) included between the left-handed material and $\mathrm{Au}$. Clear evidence of ultra-high sensitivity and specific behaviors are shown on the profiles of confined electromagnetic modes, depending on the incident angle, $\theta_{1}$ of an excitation light, which has not been yet observed in metal-insulator-metal structures.

This paper is organized as follows. First, we describe the essential formulas of electromagnetic fields that propagate within the configuration under study and the enhancement factors (EFs). Second, we present the obtained results with significant interpretations. Finally, the paper ends with conclusions. The transfer matrix method (TMM), leading to simulate the p-reflectance profile, is repeated in the appendix section.

\section{Field equations near flat interfaces of an SPR sensor}

The multilayer structure under study is schematically illustrated in Fig. 1. The one dimensional (1D) planar nanocavity, deposited on a $\mathrm{SiO}_{2}$ glass prism, is made up of a dielectric layer bounded by two active materials, LHM and Au. In the present work, the optical response was calculated for $\mathrm{Au}$ and LHM as an outermost layer. Besides, it is assumed that the in- and outer-most media extend to infinity. All the parameters of the multilayered structure are specified in Fig. 1. To create SP' mode, a continuous transverse magnetic (TM)-polarized light (under incident angle, $\theta_{1}$ ) of fixed wavelength, $\lambda$, illuminates the multilayer structure through a $\mathrm{SiO}_{2}$ glass prism.

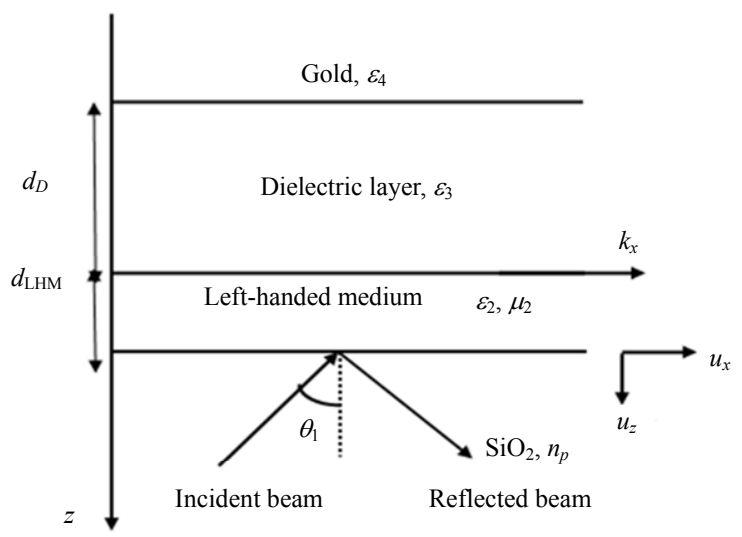

Fig. 1 Multilayer configuration under study: an LHM layer $\left(\varepsilon_{2}, \mu_{2}, d_{\mathrm{LHM}}\right)$, a dielectric layer $\left(\varepsilon_{3}, d_{D}\right)$, and an Au-bulk $\varepsilon_{\mathrm{Au}}=\varepsilon_{4}$, which are stacked along $z$-direction on a $\mathrm{SiO}_{2}$ glass prism or RI, $\left(n_{p}=\sqrt{\varepsilon_{p}}\right)$. 
Thus, assume the structure of Fig. 1, being oriented in $z$-axis, the incident beam, under the angle, $\theta_{1}<\theta_{\text {cr }}$ (critical angle), is partially reflected and transmitted on either side of the interfaces. So, the explicit equations of all the electric fields propagating in each medium at the $\mathrm{z}$-coordinate of the SPR approach: prism, LHM, dielectric layer, and $\mathrm{Au}$, can be written as the following form:

$$
E_{p}=\left\{E_{1 x}\left(\begin{array}{c}
1 \\
0 \\
k_{x} / k_{z 1}
\end{array}\right) \mathrm{e}^{\mathrm{i}\left\{k_{x} x+k_{z 1}\left[z-\left(d_{\mathrm{LHM}}+d_{D}\right)\right]\right\}}\right\} \mathrm{e}^{-\mathrm{i} \omega t}
$$

for $z \geq d_{\mathrm{LHM}}+d_{D}$, where $E_{1 x}$ denotes the electric field amplitude along the $x$-axis. Here, $k_{x}=\frac{2 \pi}{\lambda} \sqrt{\varepsilon_{p}} \sin \theta_{1}$ is the wave vector component along the $x$-axis in the prism, and $k_{z j}=\frac{2 \pi}{\lambda}\left(\varepsilon_{i} \mu_{j}-\varepsilon_{p} \sin ^{2} \theta_{1}\right)^{1 / 2}$ denotes the wave vector component along the $z$-axis in each layer of the structure indexed, $j$ with, $j=1,2,3$, and 4 , respectively:

$$
E_{\text {LHM }}=m\left\{E_{1 x}\left(\begin{array}{c}
1 \\
0 \\
k_{x} / k_{z 2}
\end{array}\right) \mathrm{e}^{\mathrm{i}\left[k_{x} x+k_{z 2}\left(z-d_{\mathrm{LHM}}\right)\right]} \mathrm{e}^{-\mathrm{i} \omega t}\right\}
$$

for $d_{\mathrm{LHM}} \geq z \geq d_{D}$, with

$$
m=\frac{\left(1-r_{12}\right)\left(1-r_{23}\right)}{\mathrm{e}^{-\mathrm{i}_{22} d_{\mathrm{LHM}}}-r_{23} \mathrm{e}^{\mathrm{i} k_{22} d_{\mathrm{LHM}}}}
$$

and

$$
E_{D}=m^{\prime}\left\{E_{1 x}\left(\begin{array}{c}
1 \\
0 \\
k_{x} / k_{z 3}
\end{array}\right) \mathrm{e}^{\mathrm{i}\left[k_{x} x+k_{z 3}\left(z-d_{D}\right)\right]} \mathrm{e}^{-\mathrm{i} \omega t}\right\}
$$

for $d_{D} \geq z \geq 0$, with

$$
m^{\prime}=\frac{\left(1-r_{12}\right)\left(1-r_{23}\right)\left(1-r_{34}\right)}{\left(\mathrm{e}^{-\mathrm{i}_{21} d_{\text {LHM }}}-r_{23} \mathrm{e}^{\mathrm{i} k_{21} d_{\text {LHM }}}\right)\left(\mathrm{e}^{-\mathrm{i}_{23} d_{\text {LHM }}}-r_{34} \mathrm{e}^{\mathrm{i} \mathrm{i}_{23} D}\right)}
$$

and

$$
r_{12}=\frac{1-b_{1}}{1+b_{1}}, r_{23}=\frac{1-b_{2}}{1+b_{2}}, r_{34}=\frac{1-b_{3}}{1+b_{3}}
$$

are the reflection coefficients of the structure interfaces.

$$
E_{A u}=m^{\prime}\left\{E_{1 x}\left(\begin{array}{c}
1 \\
0 \\
k_{x} / k_{z 4}
\end{array}\right) \mathrm{e}^{\mathrm{i}\left(k_{x} x+k_{z 4} z\right)}\right\} \mathrm{e}^{-\mathrm{i} \omega t}
$$

for $z \leq 0$, with

$$
\begin{gathered}
b_{1}=\frac{k_{z 2} \varepsilon_{p}\left(1-\mathrm{e}^{2 \mathrm{i} k_{z 2} d_{\mathrm{LHM}}}\right)}{k_{z 1} \varepsilon_{\mathrm{LHM}} \mu_{\mathrm{LHM}}\left(1+\mathrm{e}^{2 \mathrm{i} k_{22} d_{\mathrm{LHM}}}\right)} \\
b_{2}=\frac{k_{z 3} \varepsilon_{\mathrm{LHM}}\left(1-\mathrm{e}^{2 \mathrm{i}_{z 3} d_{D}}\right)}{k_{z 2} \varepsilon_{3}\left(1+\mathrm{e}^{2 \mathrm{i} k_{z 3} d_{D}}\right)}
\end{gathered}
$$

and $b_{3}=\frac{\varepsilon_{3} k_{z 4}}{\varepsilon_{4} k_{z 3}}$.

In a similar form of (1)-(4), the associated magnetic fields $B$ propagating through the proposed four-layer sensor, can be easily determined by the usual Maxwell equation:

$$
\nabla \times E+\frac{\partial B}{\partial t}=0 .
$$

The above set of (1)-(4) is the starting point to carry out a comprehensive analysis on the propagation of the guided resonant modes according to both directions, $u_{x}$ and $u_{z}$, when a monochromatic wave irradiates the structure under an incidence angle $\theta_{1}$

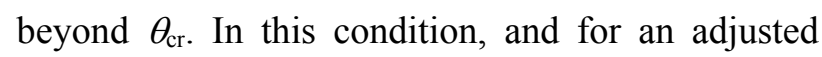
LHM layer thickness, $d_{\mathrm{LHM}}$, most of the incident energy is absorbed by the layered materials and generates SPs at the boundary LHM-dielectric layer interface. As indicated in the example geometry of Fig. 1, the supported SP modes will be studied in the two possible arrangements between LHM and $\mathrm{Au}$ separated by a dielectric layer (sensing medium). Thus, in our theoretical simulation, the chosen parameters of the active material layers are described by the complex permittivity, $e_{\mathrm{Au}}(1)=$ $-21.3+\mathrm{i} 1.35$ for gold, which is taken from [31], and $\varepsilon_{2}(\lambda)=-33.5, \mu_{2}(\lambda)=-11$ for LHM [32], which are evaluated at operating wavelength of $\lambda=738 \mathrm{~nm}$. Refractive indices for $\mathrm{SiO}_{2}$-glass prism and sensing medium are $n_{p}=\sqrt{\varepsilon_{p}}=1.57$ and $n_{3}=\sqrt{\varepsilon_{3}}=1.46$, respectively.

According to the $x z$-plane, the resulting interfacial modes, selected along $u_{x}$ and $u_{z}$ directions, can be described through the following longitudinal and perpendicular confinement factors 
as follows, respectively:

$$
\begin{aligned}
& f_{\|}\left(\theta_{1}\right)=\frac{\left|E_{\mathrm{Au}} u_{x}\right|_{z=0}}{\left|E_{p} u_{x}\right|_{z=d_{D}}}=m^{\prime} \\
& f_{\perp}\left(\theta_{1}\right)=\frac{\left|E_{A u} u_{z}\right|_{z=0}}{\left|E_{p} u_{z}\right|_{z=d_{D}}}=\frac{k_{z 1}}{k_{z 4}} m
\end{aligned}
$$

whose characteristics, therefore, are considered for the analytical resolution in terms of sensitivity (extracted from angular reflectance spectra) of the proposed SPR sensor on both the thicknesses of LHM $\left(d_{\mathrm{LHM}}\right)$ and dielectric layer $d_{D}$.

\section{Physics of surface plasmons}

Surface plasmons are basically collective oscillating charges that propagate along a thin metallic layer bounded by a dielectric medium with electric field decaying exponentially, i.e., the evanescent character, in both the media. Otherwise, SPs occur when an incident p-polarized light of a given wavelength strikes the metallic surface at an angle, $\theta_{1}>\theta_{\text {cr }}$ through the coupling prism (or metallic grating). Under these conditions, the light reflectivity from the prism base reduces to the minimum due to the plasmonic absorption mechanism. The position of this particular angle, $\theta_{1}$, called the angular resonance of SP and the width at $50 \%$ of the reflected light profile, can be substantially affected with little variation in the optical constants of associated media of the proposed device. This effect is due to the presence of the evanescent field surrounding the active metamaterial (LHM). So, due to these effects, it should be necessary to investigate the sensitivity by combining different electromagnetic properties of conducting materials as LHM and $\mathrm{Au}$ with dielectric substrates. It is to be mentioned here that the choice of $\mathrm{Au}$ comes from the fact that it presents favorable properties over other metals.

\section{Results and discussion}

First, to investigate the potential of the proposed SPR nanocavity formed with a dielectric layer of RI
$\left(n_{D}=\sqrt{\varepsilon_{D}}\right)$ and surrounded by a LHM layer $\left(e_{2}\right.$, $m_{2}$ ) and a bulk-LHM, we plot the reflectance curve versus incidence angle, as shown in Fig. 2. This interfacial response is calculated from the transfer matrix method (TMM) which was previously detailed elsewhere [22].

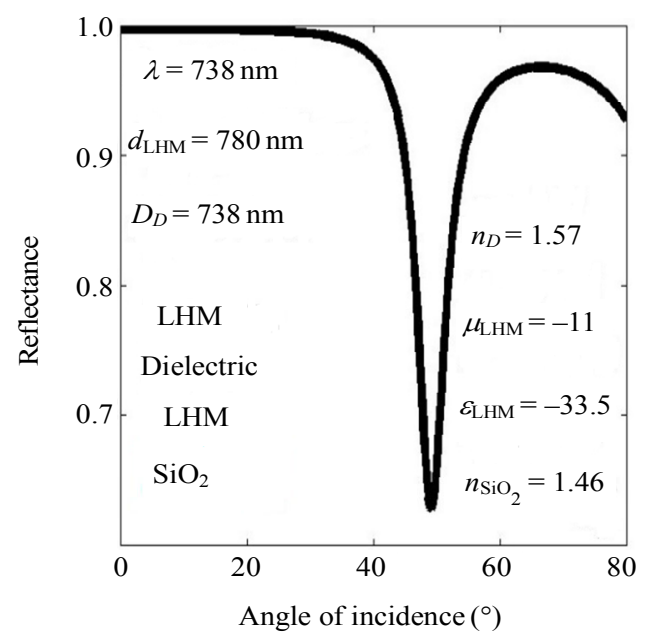

(b)

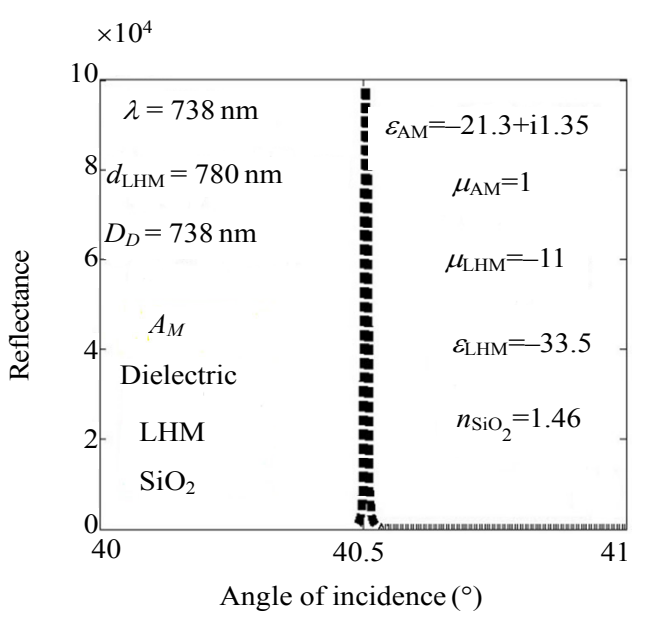

(b)

Fig. 2 Calculated angular P-reflectance spectra investigated from the samples: (a) prism-LHM layer- dielectric gap layer-LHM bulk, and (b) prism-LHM-dielectric gap layer-Au bulk. The parameter conditions are specified in the inset of the figures.

The formalism of TMM, applied to the proposed SPR nanocavity with a prism-coupler, is repeated in the appendix section of the paper. For the stacking shown in the inset of Fig.2(a), by considering the thicknesses, $780 \mathrm{~nm}$ and $330 \mathrm{~nm}$ of LHM layer and dielectric, respectively, the (1D)-nanocavity exhibits a single SPR mode excited at $49.23^{\circ}$. These 
optimized thicknesses are necessary to achieve the lowest point of the minimum p-reflectance curve of $62 \%$. For this resolved peak-SPR mode in the angular p-reflectance curve, the estimation of quality factor $Q=\theta_{\mathrm{SPR}} / \Delta \theta_{0.5}$ reaches the value of $12\left(\Delta \theta_{0.5}=5.58^{\circ}\right)$. In the case, where $\mathrm{Au}$ is taken as an outermost layer, [see Fig.2(b)], the p-reflectance curve shows the existence of a single peak-SPR mode at the angle of $\theta_{\mathrm{SPR}}=40.51^{\circ}$ whose intensity achieves a maximum value of $\sim 9.85 \times 10^{4}$. From a systematic comparison between the results of Figs. 2(a) and 2(b) in terms of the reflectance intensities, it means that $\mathrm{Au}$, with a loss tangent angle 0.063 as an outermost layer when combined with the LHM layer, causes a substantial optical amplification of an extremely narrow full width at half maximum (FWHM). The resonant modes sustained by the interfaces within the dielectric layer (sensing layer) can be coupled to each other and make more energy stored on the outermost medium $(\mathrm{Au})$. This effect, being resulted from the optical coupling process generated between electromagnetic modes inside the designed (1D)-nanocavity, has been recently observed experimentally by Hayashi et al. [33] and Goswami et al. [34] in other variant SPR systems. According to the above quantitative characteristics related to

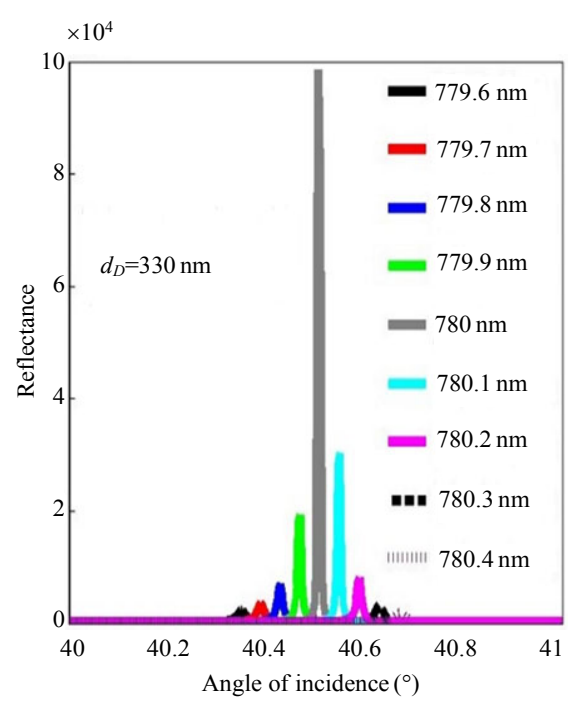

(a) the SP' excitation, the proposed device exhibits a significant application as a tunable filter.

Next, we discuss theoretically the effects induced in the resonance (ATR) intensity on both the thicknesses of LHM, $d_{\text {LHM }}$ and the dielectric layer, $d_{D}$. The structure being considered here concerns the arrangement as: $\mathrm{SiO}_{2}$ prism/LHM $\left(d_{\text {LHM }}\right) /$ dielectric gap $\left(d_{D}\right) /$ Au-bulk. As a result, with a fixed thickness, $d_{D}=330 \mathrm{~nm}$ [see Fig. 3(a)], by tuning the thickness, $d_{\text {LHM }}$ from 779.6 $\mathrm{nm}$ to $780.4 \mathrm{~nm}$, a dominant amplification of $\sim 9.85 \times 10^{4}$ arises at $40.51^{\circ}$ for $d_{\mathrm{LHM}}=780 \mathrm{~nm}$. Taking this condition, the above amplification value may be further optimized [see Fig. 3(b)] by increasing against the thickness $d_{D}$ around the range of $329 \mathrm{~nm}$ to $333 \mathrm{~nm}$. The p-reflectance peak of the exhibited SPR mode gets a value in the order of $2.13 \times 10^{6}$ estimated at the resonance angle of $40.74^{\circ}$. Such an optimization fulfilling to the condition, $d_{D} /\left(d_{D}+d_{\text {LHM }}\right) \sim 0.3$ leads to highlight that the thickness of the LHM layer, and the one of the dielectric layer of a fixed RI, $n_{D}$, plays an essential way for producing a sharper optical amplification that can be exploited in an SPR sensing purpose. It is worth noting that the effect on RI, $n_{D}$ of the dielectric layer, was discussed in our previous work [22].

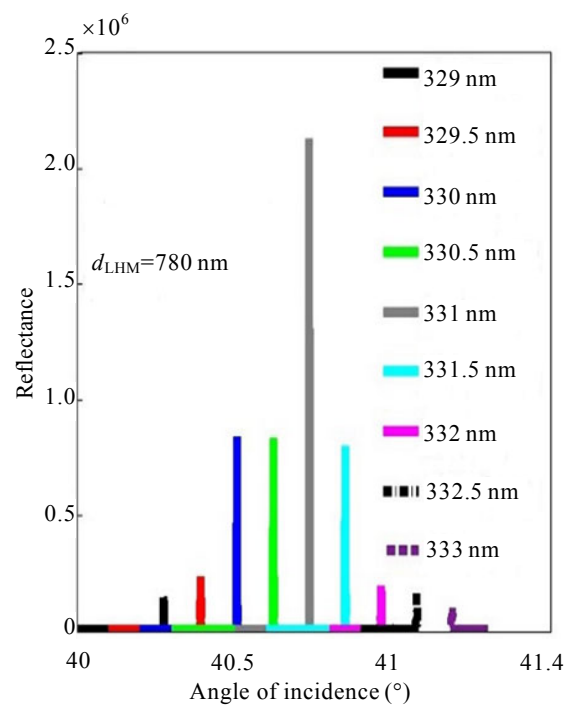

(b)

Fig. 3 Angular p-reflectance spectra for the arrangement prism/LHM/Dielectric layer/Au, when changing (a) for different LHM layer thickness from $779.6 \mathrm{~nm}$ to $780.4 \mathrm{~nm}$ with a fixed dielectric layer set to be $330 \mathrm{~nm}$, and (b) for different dielectric layer thickness from $329 \mathrm{~nm}$ to $333 \mathrm{~nm}$ with a fixed LHM thickness set to be $780 \mathrm{~nm}$. 
In this part, we put now emphasis on the characteristics evaluated on the enhancement factors (EFs) curves, $f_{\perp}$ and $f_{\|}$previously defined in Figs. 7(a) and 7(b). Therefore, in the optimized condition of Fig. 3(a), i.e., with $d_{D}=$ $330 \mathrm{~nm}$ and $d_{\mathrm{LHM}}=780 \mathrm{~nm}$, the angular dependencies of $f_{\perp}$ and $f_{\|}$in the large angular range from $33^{\circ}$ to $73^{\circ}$ are depicted in Figs. 4(a) and 4(b), respectively. On the angular profile of each curve, the total thickness, $d=\left(d_{D}+d_{\mathrm{LHM}}\right)=1110 \mathrm{~nm}$, gives the ability of generating simultaneously two sharper confined modes (associated to the supported SPs) at the resonance angles $33.83^{\circ}$ (first-mode), and $72.22^{\circ}$ (second-mode). Moreover, it should be noted that the two EFs' profiles through the structure present the same stop-band defined between the successive confined modes. The narrowest peak intensities of these confined modes for $f_{\|}$are slightly different, i.e., $0.8 \times 10^{6}$ and $1.35 \times 10^{6}$, except for the ones of $f_{\perp}$, there is rather a notable difference, $3.09 \times 10^{6}$ and $0.15 \times 10^{6}$. So, the multilayer structure: $\mathrm{SiO}_{2}$ prism/LHM $(780 \mathrm{~nm}) /$ dielectric gap $(330 \mathrm{~nm}) /$ Au-bulk sustains a giant amplification as reported in Fig. 2(b), and consequently, the structure characterized by the highest EFs is very promising to develop bio-sensing applications. For a comparison, recently, Sekkat et al. [24] proposed $\mathrm{Ag} /$ Cytop interfaces as a plasmonic planar structure leading to achieving a giant amplification $\sim 1.3 \times 10^{6}$, which remains much less than the one we obtained here.

Following the above analysis, we finally focus on simulating the relationships between the angular resonances, $\theta_{\mathrm{SPR}}$ with the variation of thicknesses, $d_{D}$ and $d_{\text {LHM }}$ of the EFs intensities as shown in Fig. 4. Thus, based on typical curves as depicted in Fig. 4(a), when we consider the thickness, $d_{D}$ fixed at $330 \mathrm{~nm}$, it is observed that the resonance condition, $\theta_{\mathrm{SPR}}$ of each mode in the EF curve of $f_{\perp}$ increases sensitively with an increase in $d_{\text {LHM }}$ from $779.6 \mathrm{~nm}$ to $780.4 \mathrm{~nm}$ [see Figs. 5(a) and 5(b)]. From these data, the observed change in SPR angle $\theta_{\mathrm{SPR}}$ as a function of the LHM thickness, $d_{\mathrm{LHM}}$, can be numerically predicted as:

$$
\begin{aligned}
\theta_{\mathrm{SPR}, \perp}\left(d_{\mathrm{LHM}}\right)= & 1.1195 d_{\mathrm{LHM}}^{3}-2623.9 d_{\mathrm{LHM}}^{2}+ \\
& 2.05 \times 10^{6} d_{\mathrm{LHM}}-5.34 \times 10^{8} \pm 0.029^{\circ} .
\end{aligned}
$$

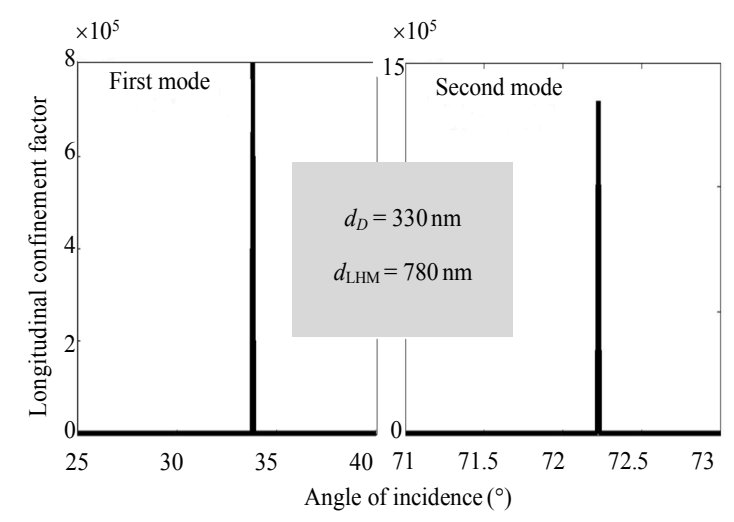

(a)

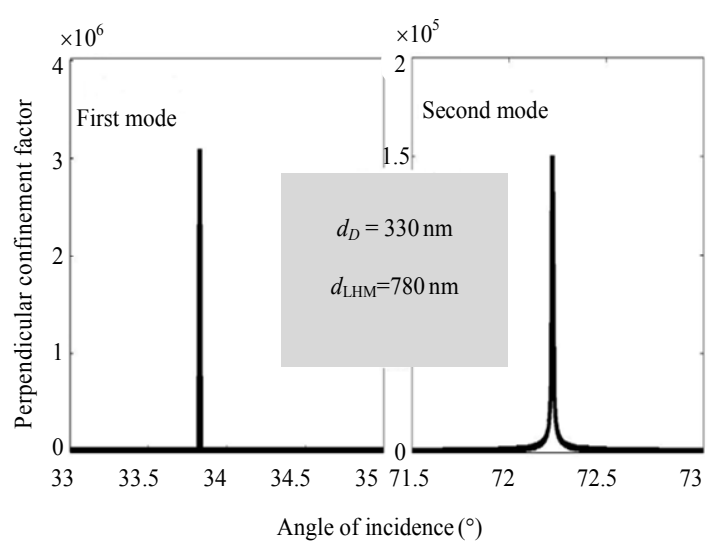

(b)

Fig. 4 Angular dependency of the interface' modes displayed on (a) the perpendicular and (b) the longitudinal confinement factors, $f_{\perp}$ and $f_{\|}$in the arrangement: prism-LHM-dielectric gap layer-Au outermost medium, with an LHM layer set to be $780 \mathrm{~nm}$., and dielectric layer is set to be $330 \mathrm{~nm}$. The other medium parameters are the same as those reported in the inset of Fig. 2(b).

For the same purpose, by considering the thickness $d_{\mathrm{LHM}}$ fixed at $780 \mathrm{~nm}$, the resonance condition $\theta_{\mathrm{SPR}}$ of each mode in the EF curve of, $f_{\wedge}(\theta)$ increases linearly with an increase in the thickness $d_{D}$ from $329 \mathrm{~nm}$ to $333 \mathrm{~nm}$ [see Figs. 5(c) and 5(d)]. Therefore, this dependency, in this condition, can be analytically predicted from the following form:

$$
\theta_{\mathrm{SPR}, \perp}\left(d_{D}\right)=\left(0.17867 \times d_{D}-25.13^{\circ}\right) \pm 0.0078^{\circ}
$$




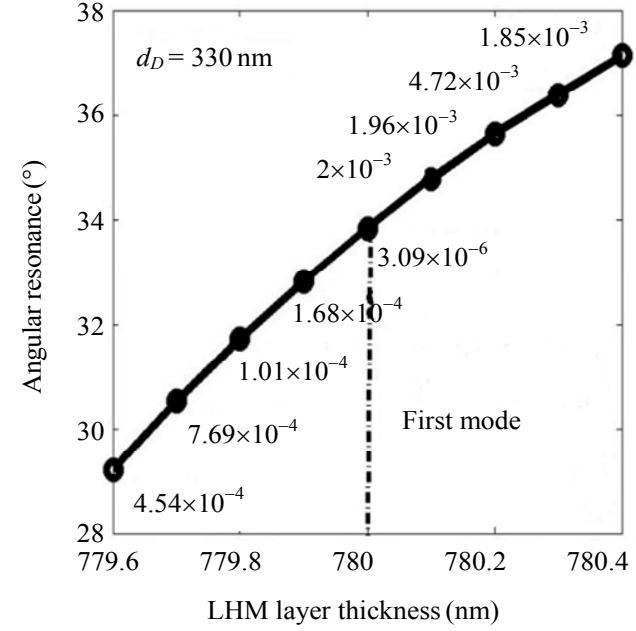

(a)

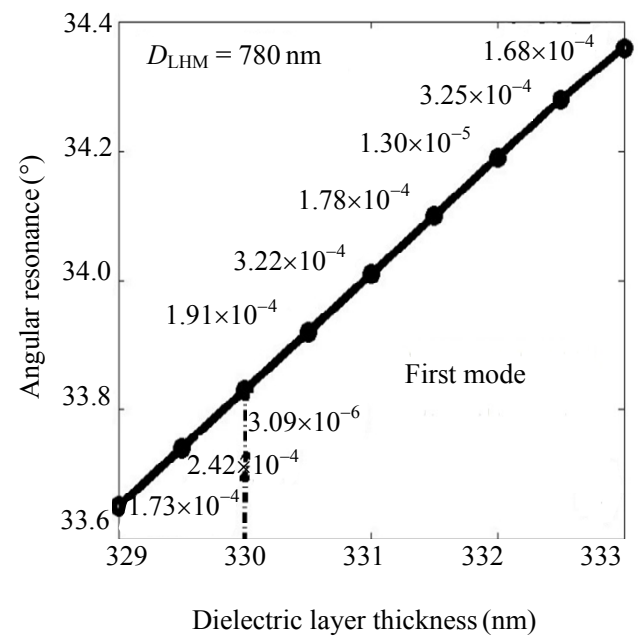

(c)

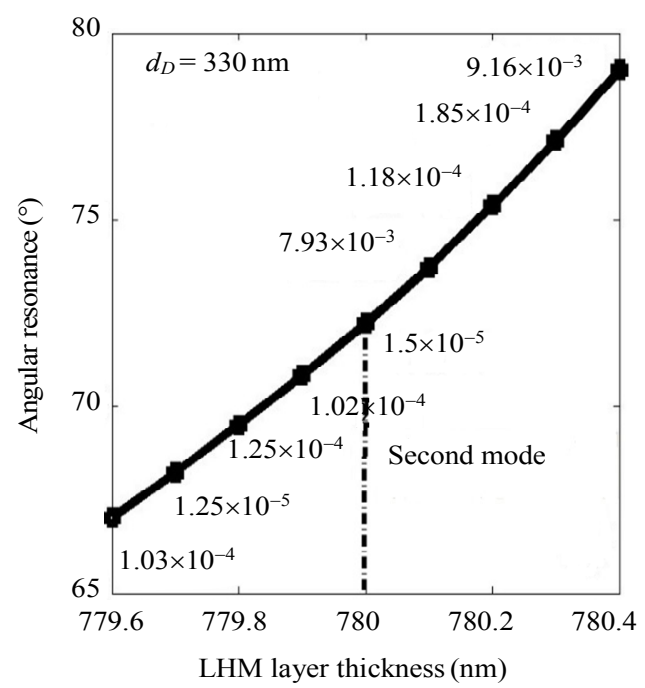

(b)

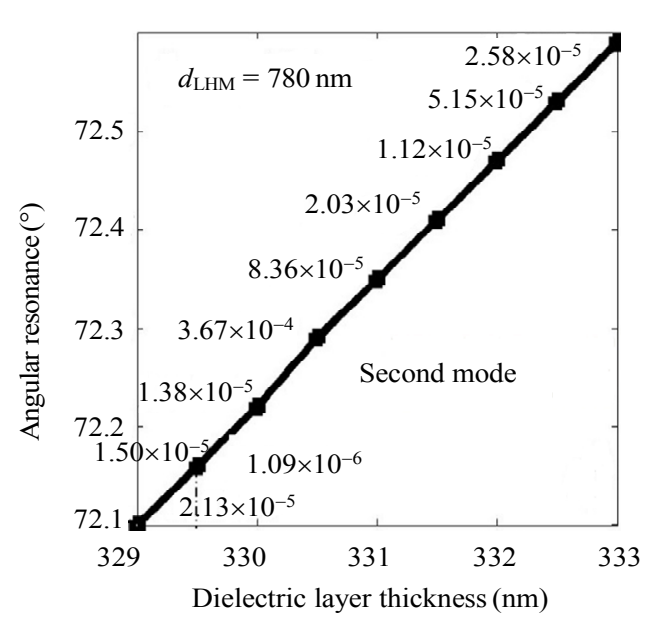

(d)

Fig. 5 Evolution in the SPR angle for: (a) the first-confined mode, (b) the second-confined mode displayed on the perpendicular enhancement factor, $f_{\perp}$ under the change of the LHM thickness with a fixed dielectric layer, $d_{D}=330 \mathrm{~nm}$, (c) first-confined mode, and (d) second-confined mode displayed on the perpendicular enhancement factor, $f_{\perp}$ under the change of the dielectric layer thickness with a fixed, metamaterial layer, $d_{\mathrm{LHM}}=780 \mathrm{~nm}$.

A similar study has been conducted on the evolution of each mode in the EF curve of $\mathrm{f}_{/ /}$versus $\mathrm{d}_{\mathrm{LHM}}$ and $\mathrm{d}_{\mathrm{d}}$. By taking into account typical curves as shown in Fig. (4), the extracted data are represented in Figs. 6(a)-6(d). For the two cases considered separately, it is observed that for each EF' mode of $f_{\|}$, their respective resonance condition increases with an increase in either $d_{\mathrm{LHM}}$ or $d_{D}$. Thus, for each condition, such a dependency in the SPR condition of $f_{/ /}$curve versus $d_{L H M}$ or $d_{D}$ can be predicted, respectively, by the following relations:

$$
\begin{aligned}
\theta_{\mathrm{SPR}, \|}\left(d_{\mathrm{LHM}}\right)= & 1.1195 d_{\mathrm{LHM}}^{3}-2801.2 d_{\mathrm{LHM}}^{2}+ \\
& 2.1882 \times 10^{6} d_{\mathrm{LHM}}-5.6979 \times 10^{8} \\
\theta_{\mathrm{SPR}, \|}\left(d_{D}\right)= & 0.1796 \times d_{D}-25 .
\end{aligned}
$$

In Figs. 5 and 6, the reported values on these curves represent the achieved intensities of $\mathrm{EF}$ modes according to the relative values of the thicknesses $d_{D}$ and $d_{\mathrm{LHM}}$. 


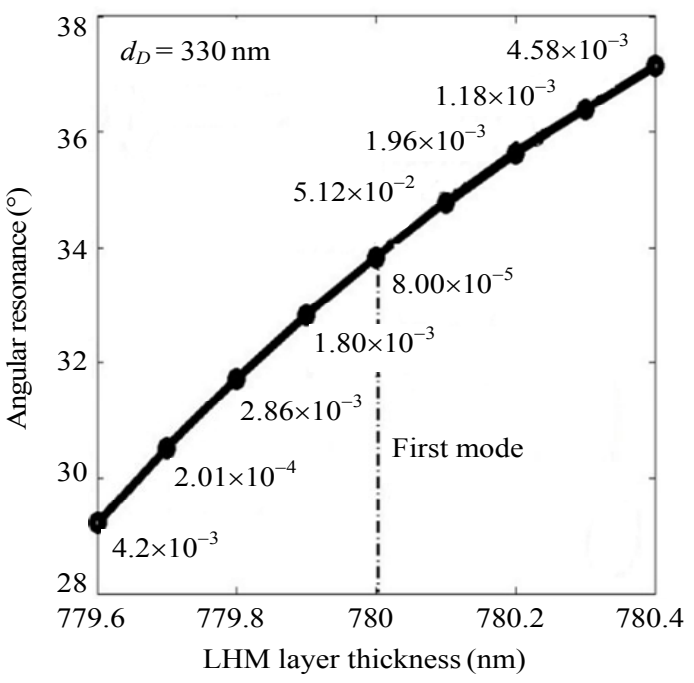

(a)

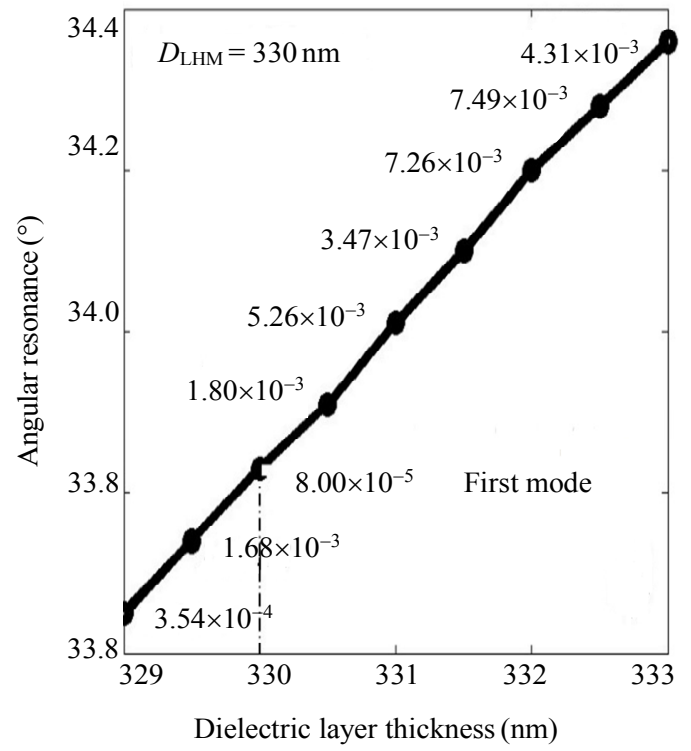

(c)

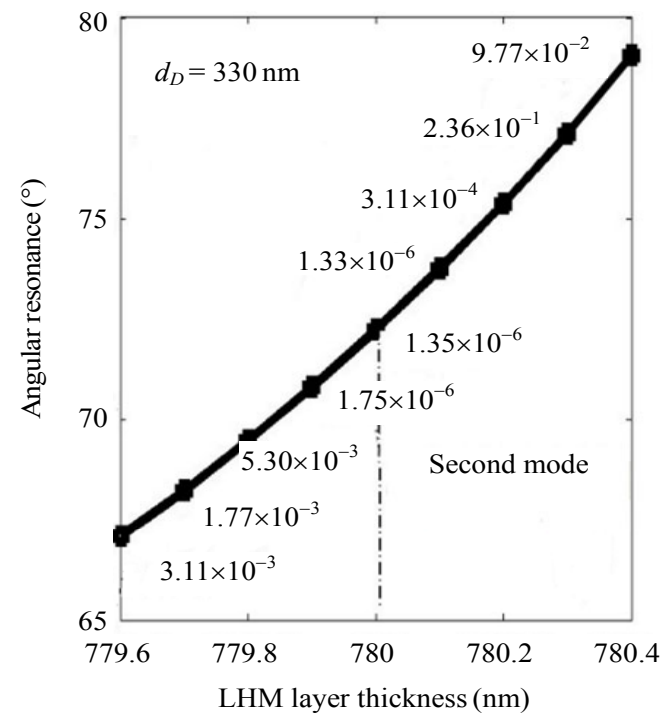

(b)

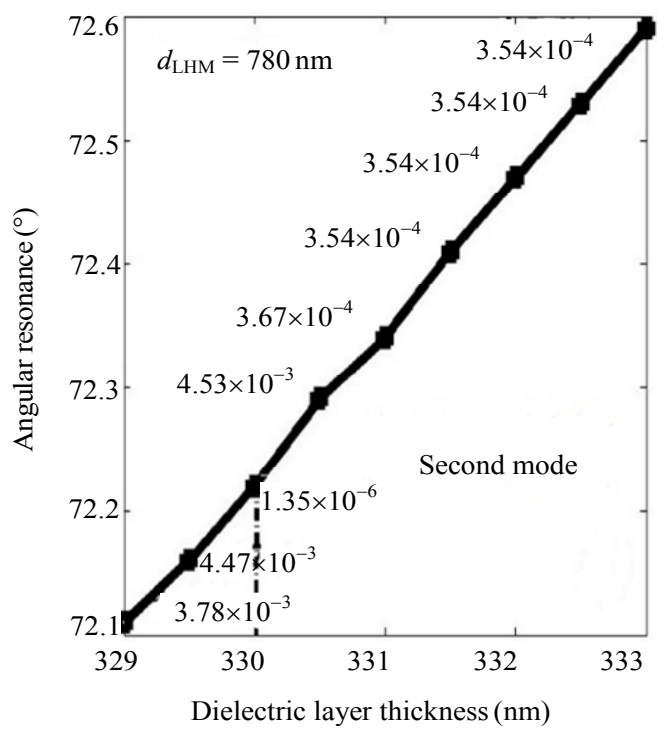

(d)

Fig. 6 Evolution in the SPR angle for: (a) first-confined mode and (b) second-confined mode displayed on the parallel enhancement factor $f_{\|}$under the change of LHM thickness with a fixed $d_{D}=330 \mathrm{~nm}$, (c) first-confined mode, and (d) second-confined mode displayed on the parallel enhancement factor $f_{\|}$under the change of dielectric gap with a fixed thickness, $d_{\mathrm{LHM}}=780 \mathrm{~nm}$.

\section{Conclusions}

In this paper, a plasmonic sensor, with an active LHM layer of simultaneous negative permittivity and permeability, stacked on a dielectric layer and $\mathrm{Au}$, has been investigated theoretically. This SPR sensor, based on gold $(\mathrm{Au})$ taken as an outermost medium, has the capability of exhibiting substantial electromagnetic modes confined on the structure' interfaces. However, the resulting optical amplification predicted on both the longitudinal and perpendicular factors due to the existence of resonant modes with narrower angular widths, depends critically on the ratio evaluated between the structure' thicknesses. It has been observed that the characteristics (peak intensity, resonance angle, and line width) of the SPR modes probed in an angular interrogation method can be highly controlled on the change of the dielectric gap thickness of fixed refractive index. Based on the 
electromagnetic calculations, the giant optical amplification $\sim 3.09 \times 10^{6}$ of exhibited mode on the interfaces of the proposed device, is highly sensitive to the ratio defined between the structure thicknesses $d_{D} /\left(d_{D}+d_{\text {LHM }}\right)$ and it can be constituted as a new limit which is even greater than the one of conventional SPR sensors for developing photonic applications.

\section{Appendix A}

Since the proposed planar SPR sensor displayed in the arrangement of Fig. 1 is comprised of four layers stacked along the $z$-axis, its corresponding angular p-reflectance accordingly to the TMM is given as

$\left|R_{p}\left(\theta_{i}\right)\right|^{2}=\left|\frac{\left(M_{11}+M_{12} q_{4}\right) q_{1}-\left(M_{21}+M_{22} q_{4}\right)}{\left(M_{11}+M_{12} q_{4}\right) q_{1}+\left(M_{21}+M_{22} q_{4}\right)}\right|^{2}$.

In above (A1), $M_{i j}$ elements, for $i=j=1,2$, satisfy the following expressions:

$$
\begin{aligned}
& M_{11}=\left(1+\frac{q_{3}}{q_{2}} \tan \beta_{2} \tan \beta_{3}\right) \prod_{k=2}^{3} \cos \beta_{k} \\
& M_{12}=-\mathrm{i}\left(\frac{1}{q_{3}}+\frac{1}{q_{2}} \frac{\tan \beta_{2}}{\tan \beta_{3}}\right) \cos \beta_{2} \sin \beta_{3} \\
& M_{21}=-\mathrm{i}\left(q_{2}+q_{3} \times \frac{\tan \beta_{3}}{\tan \beta_{2}}\right) \sin \beta_{2} \cos \beta_{3} \\
& M_{22}=\left(1+\frac{q_{2}}{q_{3}} \tan \beta_{2} \tan \beta_{3}\right) \prod_{k=2}^{3} \cos \beta_{k}
\end{aligned}
$$

with

$$
\left\{\begin{array}{l}
\beta_{2}=\frac{2 \pi d_{\mathrm{LHM}}}{\lambda}\left(\varepsilon_{2} \mu_{2}-\varepsilon_{p} \sin ^{2} \theta_{1}\right) \\
\beta_{3}=\frac{2 \pi d_{D}}{\lambda}\left(\varepsilon_{3}-\varepsilon_{p} \sin ^{2} \theta_{1}\right)
\end{array}\right.
$$

where $i=\sqrt{-1}$ is the imaginary complex. The terms $q_{n}$ for $n=1,2,3,4$ corresponding to the wave vector compounds along the $z$-axis in prism $\left(\mathrm{SiO}_{2}\right)$, LHM layer, sensing medium layer (dielectric), and outermost medium ( $\mathrm{Au})$, respectively, are expressed as

$$
\left\{\begin{array}{l}
q_{1}=\left(\frac{1}{\varepsilon_{p}}\right)^{1 / 2} \cos \theta_{1}, q_{2}=\frac{1}{\varepsilon_{2}}\left(\varepsilon_{2} \mu_{2}-\varepsilon_{p} \sin ^{2} \theta_{1}\right)^{1 / 2} \\
q_{3}=\frac{1}{\varepsilon_{3}}\left(\varepsilon_{3}-\varepsilon_{p} \sin ^{2} \theta_{1}\right)^{1 / 2}, q_{4}=\frac{1}{\varepsilon_{4}}\left(\varepsilon_{4}-\varepsilon_{p} \sin ^{2} \theta_{1}\right)^{1 / 2} .
\end{array}\right.
$$

Hence, to extract the performance parameters as previously discussed, in the adopted approach of four layers, p-reflectance, (A1) at the prism-active material interface is to be exploited analytically versus incidence angle $\theta_{i}$ for a fixed wavelength $\lambda$ of incident beam.

Open Access This article is distributed under the terms of the Creative Commons Attribution 4.0 International License (http://creativecommons.org/licenses/by/4.0/), which permits unrestricted use, distribution, and reproduction in any medium, provided you give appropriate credit to the original author(s) and the source, provide a link to the Creative Commons license, and indicate if changes were made.

\section{References}

[1] A. Verma, A. Prakash, and R. Tripathi, "Sensitivity enhancement of surface plasmon resonance biosensor using graphene and air gap," Optics Communications, 2015, 357: 106-112.

[2] O. Saison-fransioso, G. Lévêque, A. Akjouj, Y. Pennec, B. Djafari-rouhani, S. Szunerits, et al., "Plasmonic nanoparticules arrays for high-sensitivity sensing: a theoretical investigation," The Journal of Physical Chemistry C, 2012, 116(33): 17819-17827.

[3] M. S. Rahman, M. S. Anower, L. B. Bashar, and K. A. Rikta, "Sensitivity analysis of grapheme coated surface plasmon resonance biosensors for biosensing applications," Sensing and Bio-sensing Research, 2017, 16: 41-45.

[4] A. K. Sharma, R. Jha, and B. D. Gupta, "Fiber-optic sensors based on surface plasmon resonance: a comprehensive review," IEEE Sensors Journal, 2007, 7(8): 1118-1129.

[5] A. Shalabney, C. Khare, B. Rauschenbach, and I. Abdulhalim, "Sensitivity of surface plasmon resonance sensors based on metallic columnar thin films in the spectral and angular interrogations," Sensors and Actuators B: Chemical, 2011, 159(1): 201-212.

[6] S. Szunerits, X. Castel, and R. Boukherroub, "Surface plasmon resonance investigation of silver and gold films coated with thin indium tin oxide layers: influence on stability and sensitivity," The Journal of Physical Chemistry C, 2008, 112(40): 15813-15817. 
[7] S. A. Taya and H. M. Kullab, "Optimization of transverse electric peak-type metal clad waveguide sensor using double-negative materials," Applied Physics A, 2014, 116(4): 1841-1846.

[8] H. P. Chiang, C. W. Chen, J. J. Wu, H. L. Li, T. Y. Lin, E. J. Sanchez, et al., "Effects of temperature on the surface plasmon resonance at a metal-semiconductor interface," Thin Solid Films, 2007, 515(17): 6953-6961.

[9] B. Bouhafs, M. Benatallah, and M. Bendjebbour, "Resonant electromagnetic field distribution on doped multilayer thin film structure," Spectroscopy Letters, 2014, 47(5): 397-403.

[10] H. Deng, D. Yang, B. Chen, and C. Lin, "Simulation of surface plasmon resonance of $\mathrm{Au}-\mathrm{WO}_{3-x}$ and $\mathrm{Ag}-\mathrm{WO}_{3-x}$ nanocomposite films," Sensors and Actuators B: Chemical, 2008, 134(2): 502-509.

[11] S. Szunerits, X. Castel, and R. Boukherroub, "Preparation of electrochemical and surface plasmon resonance active interfaces: deposition of indium tin oxide on silver thin films," The Journal of Physical Chemistry C, 2008, 112(29): 10883-10888.

[12] Y. Yuan and Y. Dai, "A revised LRSPR sensor with sharp reflection spectrum," Sensors, 2014, 14(9): 16664-16671.

[13] T. Vary and P. Markos, "Propagation of surface plasmon polaritons through gradient index and periodic structures," Opto-Electronics Review, 2010, 18(4): 400-407.

[14] R. Slavik and J. Homola, "Simultaneous excitation of long and short range surface plasmons in an asymmetric structure," Optics Communications, 2006, 259(2): 507-512.

[15] L. Shen and Z. Wang, "Guided modes in a four-layer slab waveguide with dispersive left-handed material," Journal of Electromagnetic Analysis and Applications, 2010, 2(04): 264.

[16] C. Nylander, B. Liedberg, and T. Lind, "Gas detection by means of surface plasmon resonance," Sensors and Actuators, 1982, 3: 79-88.

[17] K. Lin, Y. Lu, J. Chen, R. Zheng, P. Wang, and H. Ming, "Surface plasmon resonance hydrogen sensor based on metallic grating with high sensitivity," Optics Express, 2008, 16(23): 18599-18604.

[18] J. W. Chung, S. D. Kim, R. Bernhardt, and J. C. Pyun, "Application of SPR biosensor for medical diagnostics of human hepatitis B virus (hHBV)," Sensors and Actuators B: Chemical, 2005, 111: 416-422.

[19] K. Wen, L. Yan, W. Pan, B. Luo, Z. Guo, Y. Guo, et al., "Design of plasmonic comb-like filters using loop-based resonators," Plasmonics, 2013, 8(2): 1017-1022.

[20] A. Verma, A. Prakash, and R. Tripathi, "Sensitivity enhancement of surface plasmon resonance biosensor using graphene and air gap," Optics Communications, 2015, 357: 106-112.

[21] S Pal, Y. K. Prajapati, J. P. Saini, and V. Singh, "Sensitivity enhancement of metamaterial- based surface plasmon resonance biosensor for near infrared," Optica Applicata, 2016, 46(1): 131-143.

[22] A. Cherifi and B. Bouhafs, "Potential of SPR sensors based on multilayer interfaces with gold and LHM for biosensing applications," Photonic Sensors, 2017, 7(3): 199-205.

[23] S. Hayashi, D. V. Nesterenko, A. Rahmouni, H. Ishitobi, Y. Inouye, S. Kawata, et al., "Light-tunable Fano resonance in metal-dielectric multilayer structures," Scientific Reports, 2016, 6: 33144.

[24] Z. Sekkat, S. Hayashi, D. V. Nesterenko, A. Rahmouni, S. Refki, H. Ishitobi, et al., "Plasmonic coupled modes in metal-dielectric multilayer structures: Fano resonance and giant field enhancement," Optics Express, 2016, 24(18): 20080-20088.

[25] Q. Ouyang, S. Zeng, X. Dinh, P. Coquet, and K. Yong, "Sensitivity enhancement of $\mathrm{MoS}_{2}$ nanosheet based surface plasmon resonance biosensor," Procedia Engineering, 2016, 140: 134-139

[26] H. M. Kullab, S. A. Taya, and T. M. El-Agez, "Metal-clad waveguide sensor using a left-handed material as a core layer," Journal of the Optical Society of America B, 2012, 29(5): 959-964.

[27] Q. Ouyang, S. Zeng, L. Jiang, L. Hong, G. Xu, X. Dinh, et al., "Sensitivity enhancement of transition metal dichalcogenides/silicon nanostructure-based surface plasmon resonance biosensor," Scientific Reports, 2016, 6: 28190.

[28] A. K. Sharma, "Model of a plasmonic phase interrogation probe for optical sensing of hemoglobin in blood samples," Sensing and Imaging, 2015, 16(1): 10.

[29] Q. Meng, X. Zhao, C. Lin, S. Chen, Y. Ding, and Z. Chen, "Figure of merit enhancement of a surface plasmon resonance sensor using a low-refractive-index porous silica film," Sensors, 2017, 17(8): 1846.

[30] S. A. Taya, "Slab waveguide with air core layer and anisotropic left-handed material claddings as a sensor," Opto-Electronics Review, 2014, 22(4): 252-257.

[31]A. D. Rakic', A. B. Djurisic', J. M. Elazar, and M. L. Majewski, "Optical properties of metallic films for vertical-cavity optoelectronic devices," Applied Optics, 1998, 37(22): 5271-5283.

[32]S. M. Xiao, V. P. Drachev, A. V. Kildishev, X. J. Ni, U. K. Chettiar, H. K. Yuan, et al., "Loss-free and active optical negative-index metamaterials," Nature, 2010, 466(7307): 735.

[33] S. Hayashi, D. V. Nesterenko, and Z. Sekkat, "Waveguide-coupled surface plasmon resonance sensor structures: Fano lineshape engineering for ultrahigh-resolution sensing," Journal of Physics D: Applied Physics, 2015, 48(32): 325303.

[34] N. Goswami, A. Saha, and A. Ghosh, "Optical amplification with surface plasmon resonance and total internal reflection in gold nanostructure with BK7 parallel slab," International Journal of Chemtech Research, 2014, 7(3): 1148-1153 